\title{
Two incidental lesions: a benign adrenal schwannoma and cerebral meningioma
}

\author{
P Avari1 ${ }^{1}$ A Sharma1 1 T Galliford1
}

1Department of Endocrinology, Watford General Hospital, West Hertfordshire Hospitals NHS Trust, UK

\section{INTRODUCTION}

- Adrenal schwannomas are an extremely uncommon cause of an incidentaloma, originating from the neural sheath

Schwann cells of the adrenal gland.

- We report a rare case of two incidental lesions, a benign adrenal schwannoma and cerebral meningioma

- To our knowledge, there are no cases in the literature to link de-novo adrenal schwannoma and meningioma in patients.

\section{CASE HISTORY}

- A 76 year old Caucasian gentleman presented to ED with a seizure and a community-acquired pneumonia.

- Past medical history included atrial fibrillation and ischaemic heart disease, for which he was on warfarin and bisoprolol.

- He was started on antibiotics for pneumonia. Urgent CT head revealed a left frontal lobe lesion, radiologically in keeping with a meningioma.

- As part of his work-up, CT imaging revealed an incidental left adrenal lesion, approximately $5.5 \times 4.0 \mathrm{~cm}$.

- On further assessment, he reported gaining little weight, but had no clinical signs to suggest cortisol excess. Abdomen soft with no palpable masses.
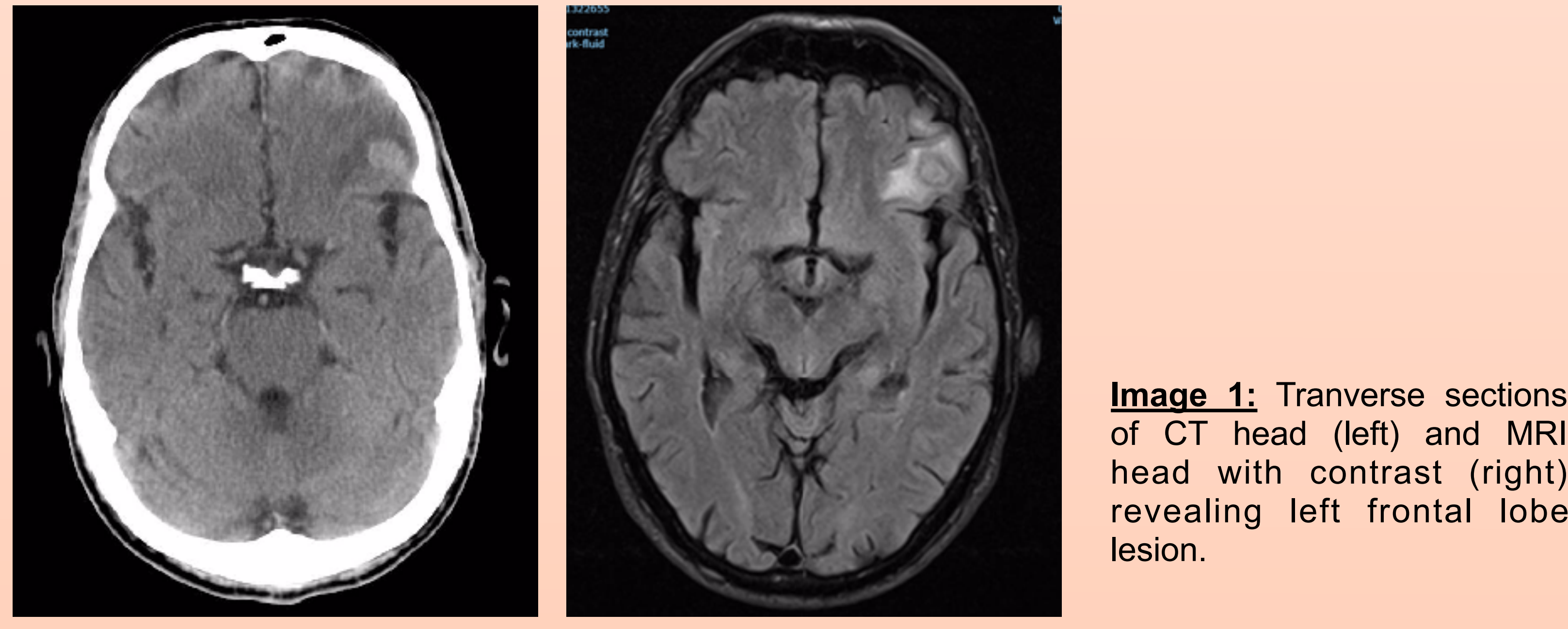

CT head (left) and MRI head with contrast (right)
revealing left frontal lobe

\section{INVESTIGATIONS}

Endocrine investigations revealed:

- Sodium $143 \mathrm{mmol} / \mathrm{L}(137-147 \mathrm{mmol} / \mathrm{L})$

- Potassium 4.4mmol/L (3.6-5mmol/L)

- Renin/aldosterone: normal

- 24-hour urinary catecholamines: normal

- 24-hour urinary cortisol: $171 \mathrm{nmol} / 24 \mathrm{rs}$ (normal)

- Overnight dexamethasone suppression test: $29 \mathrm{nmol} / \mathrm{L}$ (normal)

- Triple phase CT adrenal scan: an indeterminate solid tumour with no contrast wash-out, and features concerning for a primary adrenocortical carcinoma.
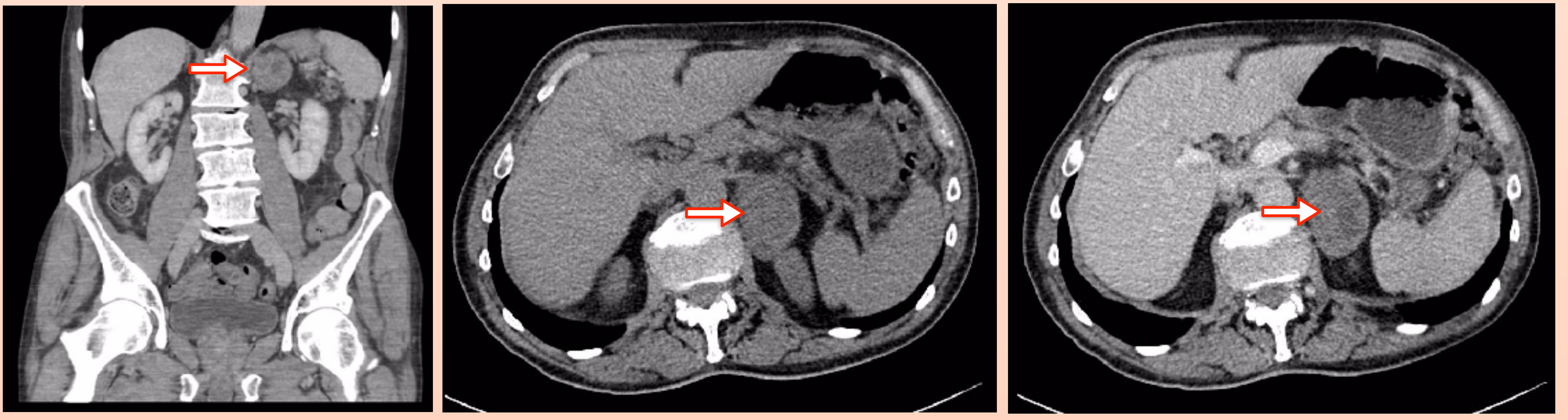

Image 2 CT adrenals: Left: Coronal image of CT abdomen depicting adrenal $5.5 \mathrm{~cm} \times 4.0 \mathrm{~cm}$ lesion (arrow). Axial views pre-contrast (middle) and 60s post
carcinoma with no contrast washout.

\section{TREATMENT}

- The case was discussed at both neurosurgical and adrenal MDTs. The neurosurgical MDT outcome was for resection of the brain tumour due to size and presentation with seizure.

- However, despite the initial presentation of a seizure, decision was made for left adrenalectomy prior to resection of the meningioma.

- Clinical priority was based on the adrenal lesion being radiologically suggestive of adrenocortical carcinoma, versus a likely benign meningioma.

- Final histology for both lesions confirmed a benign adrenal tumour consistent with schwannoma and a Grade 2 frontal lobe meningioma.

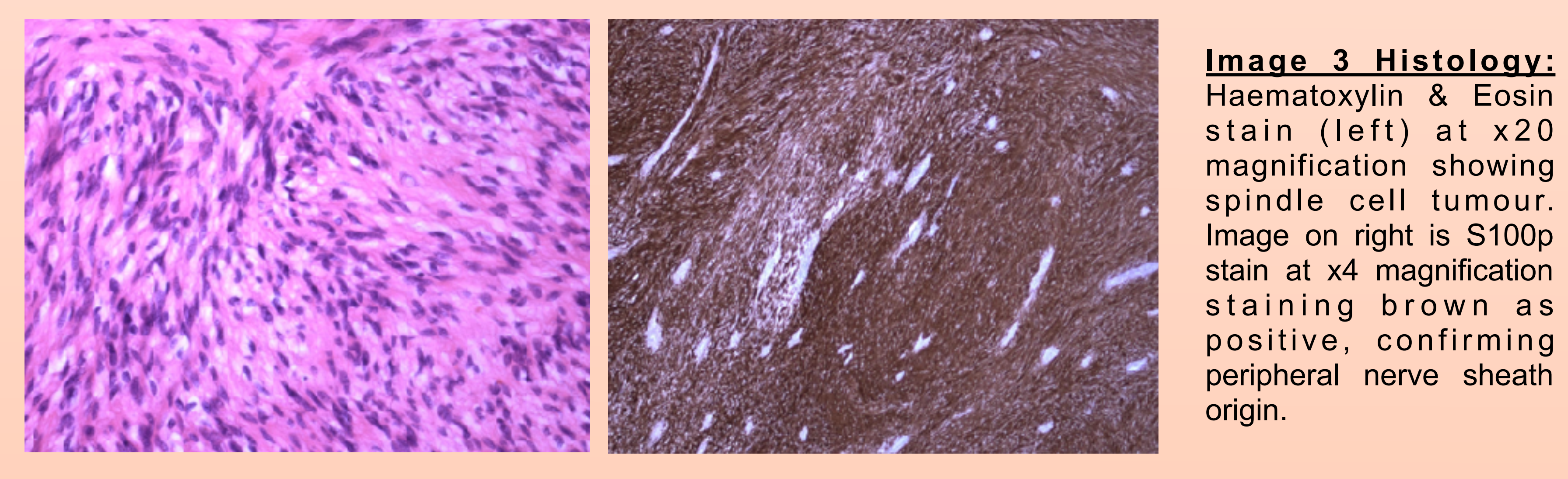

\section{DISCUSSION}

- To date, no cases of a link between de-novo adrenal schwannoma and meningioma in patients has been reported in the literature.

- Adrenal schwannomas overall are very rare tumours that are difficult to diagnose preoperatively.

- These adrenal tumours are characterized by a benign course are encapsulated and vary from firm solitary masses to fluctuant cysts. However, large masses $>4 \mathrm{~cm}$ and in the context of possible malignancy, complete laparoscopic excision is the treatment of choice.

- This case highlights the importance of multidisciplinary working to ensure expedited management in such cases.

- Awareness of benign adrenal lesions is vital for accurate pathological diagnosis to guide optimal patient management.

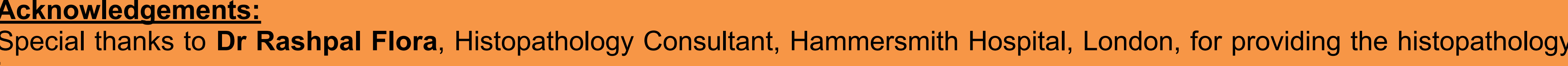
mages.

1. UWhitaker WG, Droulias C. Benign encapsulated neurilemoma: a report of 76 cases. Am Surg. 1976;42:675-678. Lee et al. Abdom Radiol (NY). Abdominal schwannomas: review of imaging findings and pathology.2017 Jul;42(7):1864-1870. 